\title{
Observing Single Pulses Over a Broad Frequency Range
}

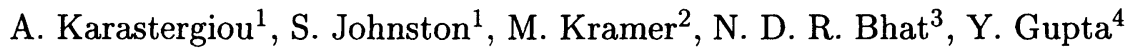 \\ ${ }^{1}$ School of Physics, University of Sydney, NSW 2006, Australia \\ ${ }^{2}$ Jodrell Bank Observatory, University of Manchester, Macclesfield, \\ Chesire SK11 9DL, UK \\ ${ }^{3}$ Arecibo Observatory, HC3 Box 53995, Arecibo, Puerto Rico, PR \\ 00612, USA \\ ${ }^{4}$ NCRA, TIFR, Pune University Campus, Ganeshkind, Pune 411007, \\ India
}

Abstract. We have obtained simultaneous observations of single pulses from the bright pulsars $\mathrm{B} 0329+54$ and $\mathrm{B} 1133+16$ at two frequencies in full polarization and a total of four frequencies in total power only. Here is a summary of our fantastic results!

\section{Total Power Results}
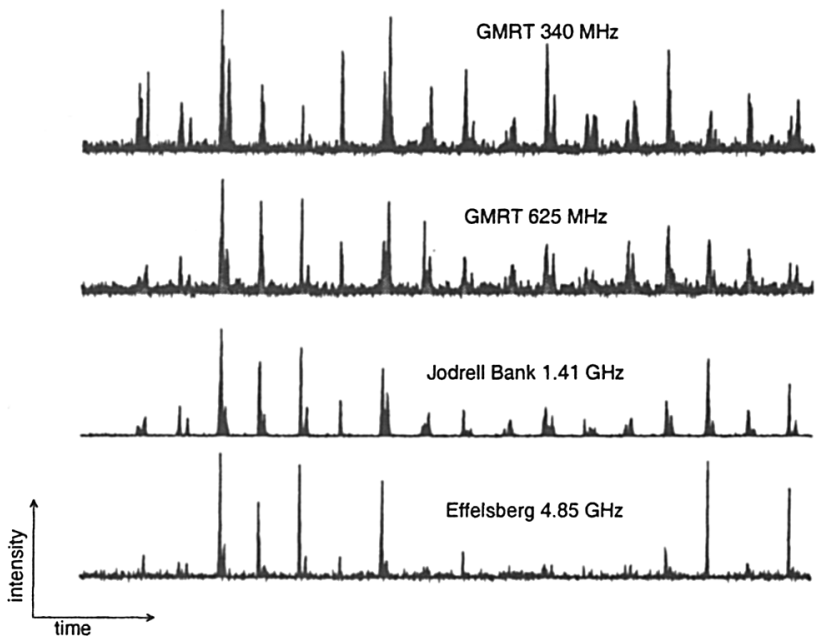

Figure 1. 17 single pulses from PSR B1133+16 in total power.

Figure 1 shows 17 single pulses from pulsar B1133+16, observed simultaneously at $340,625,1410$ and $4850 \mathrm{MHz}$ at GMRT (first two frequencies), Jodrell Bank and Effelsberg respectively. We looked at the single-pulse spectra of the individual pulse components of PSRs B0329+54 and B1133+16 after removing the effects of scintillation. For PSR B0329+54, we find that the spectral in- 
dices are different for the three components. The trailing, rather than the core, component of this pulsar has single pulses with the steepest mean spectrum. In the case of PSR B1133+16, we find that the distribution of spectral indices in the leading component is a skewed Gaussian, due to a significant population of strong pulses at the higher frequencies. These pulses constitute giant pulses in some cases at $4.85 \mathrm{GHz}$ (under the "10x the mean flux density" definition) and a study is underway to investigate whether this behavior extends to frequencies around $8 \mathrm{GHz}$ and whether these strong pulses are correlated to other phenomena such as orthogonal modes of polarization. The correlations in flux density between frequency pairs for the two pulsars are generally very good, but become weaker as the frequency separation widens. The best correlation is obtained between 600 and $1400 \mathrm{MHz}$ (Kramer et al. 2003).

\section{Polarization Results}

We have conducted polarimetric observations of the same pulsars, simultaneously observed at $1.41 \mathrm{GHz}$ at Jodrell Bank and $4.85 \mathrm{GHz}$ at Effelsberg. Especially in the case of PSR B1133+16, we took a look for the first time at the behavior of orthogonal polarization modes (OPMs) in simultaneously single pulses (Karastergiou et al. 2001). We discovered that although the position angle distribution at a given pulse phase is frequency dependent, there is a strong statistical preference for the same OPM to be stronger than its orthogonal counterpart at both frequencies (Karastergiou et al. 2002). Despite this, we find that circular polarization is not well correlated between the frequencies. A subsequent deeper investigation into the properties of circular polarization at 1.41 and $4.85 \mathrm{GHz}$ for pulsar $\mathrm{B} 1133+16$ showed that the handedness at a given pulse phase tends to be less correlated with the dominant OPM at the highest of the two frequencies (Karastergiou, Johnston \& Kramer 2003). Even at 1.41 $\mathrm{GHz}$, we do not observe the strong association between the handedness of circular polarization and the position angle seen by Cordes, Rankin \& Backer (1978) for PSR B2020+28 at lower frequencies and implied by McKinnon \& Stinebring (2000) in their method of OPM decomposition of pulse profiles. We conclude that this association is frequency dependent, which gives a new dimension to the problem of the origin of the elusive circular polarization we observe.

Simultaneous observations at four frequencies in full polarization have already taken place and exciting new results are on their way!

\section{References}

Cordes, J. M., Rankin, J., \& Backer, D. C. 1978, ApJ, 223, 961

Karastergiou, A., et al. 2001, A\&A, 379, 270

Karastergiou, A., Kramer, M., Johnston, S., Lyne, A. G., Bhat, N. D. R., \& Gupta, Y. 2002, A\&A, 391, 247

Karastergiou, A., Johnston, S., \& Kramer, M. 2003, A\&A, 404, 325

Kramer, M., Karastergiou, A., Gupta, Y., Johnston, S., Bhat, N. D. R., \& Lyne, A. G. 2003, A\&A, 407, 655

McKinnon, M. M., \& Stinebring, D. R. 2000, ApJ, 529, 435 